\title{
Study of the specific detachment characteristics of HL-2A
}

\author{
Y.D.Pan ${ }^{\mathrm{a}}$ R.Schneider ${ }^{* b}$ \\ ${ }^{a}$ Southwestern Institute of Physics, P.O.Box 432, Chengdu, 610041,China \\ ${ }^{b}$ Max-Planck-Institute fur Plasmaphysik, Euratom Association, Greifswald, Germany
}

\begin{abstract}
Numerical analysis of HL-2A single null and double null divertor discharges was done using the two-dimensional edge plasma simulation code SOLPS5.0. The detachment characteristic of HL-2A is determined by the specific divertor geometry of HL-2A. Its thin divertor throats are transparent for neutrals, but still quite well baffled towards the main chamber due to the long baffle lengths. This results in momentum losses at low densities from the interaction of the streaming divertor plasma with this neutral cushion. Therefore, a strong deviation from the common 2-point scaling is observed if local parameters are used, similar to island divertor in stellarator. The thin divertor throats in HL-2A pose MHD shaping problems resulting in strongly enhanced main chamber losses, because a large amount of the power is lost at baffles and does not even get into the divertor.
\end{abstract}

PACS:52.55.Rk, 52.55.Fa, 52.40.-W

JNM keywords: Theory and modeling, Hydrogen, Divertor materials, Heat treatment PSI-17 Keyword: B2/EIRENE, Divertor plasma, Divertor geometry, Divertor modeling Presenting and Corresponding author: Yudong Pan Presenting and Corresponding author e-mail: panyd@swip.ac.cn, Tel.: +86-28 82850339; Fax:+86-28 82850300 


\section{Introduction}

HL-2A was rebuilt in China from the old ASDEX in the final water-cooled divertor version DVII. The HL-2A tokamak (with major radius $\mathrm{R}=1.65 \mathrm{~m}$ and minor radius $\mathrm{a}=0.4 \mathrm{~m}$ ) has a close symmetric double-null divertor. Typical operational parameters of plasma are a plasma current $\mathrm{Ip}=400 \sim 500 \mathrm{kA}$, a toroidal field $\mathrm{B}_{\mathrm{T}}=2.5 \mathrm{~T}$ and a discharge duration 5.0 s with the double-null (DN) or single-null (SN) divertor configuration.

The primary aim of the studies done so far has been the identification of the major control parameters, the exploration of the operational window and the investigation of the effect of divertor geometry. Even the simulation work is carried out almost 20 years after its construction; it still provides a well-defined path for the basic physics and can contribute such to extrapolate to next step machines. The plasma edge/divertor region is modeled with the solps5.0 plasma edge transport code package ${ }^{[1][2][3]}$. The specific closed divertor configuration of HL-2A creates many interesting features. A unique feature, which will be discussed in this paper, is the appearance of momentum losses already at very low densities and a quite unusual detachment sequence very similar to that in island divertor of a stellarator ${ }^{[4]}$.

\section{Simulation model}

The two dimensional SOLPS5.0 ${ }^{[5]}$ plasma edge modeling code package solves a set of fluid equations for electrons and an arbitrary number of ion species. A neutral fluid model is used, where Charge exchange (CX) gives rise to diffusion and ionization to the loss of neutrals. The numerical grids used in the present study are shown in Fig1. The very thin divertor structures cause problems of getting the power into divertor and onto the divertor 
plates by proper MHD shaping. Small mismatches in the MHD equilibrium directly lead to very narrow scrape-off layers limited by wall and limiter contacts resulting in large wall fluxes.

As a starting point we have taken an ohmic discharge in hydrogen. A power of $500 \mathrm{~kW}$ crossing the core interface boundary (CIB), which is a few centimeters inside the core plasma, takes into account already losses within the rest of the core region not included within the B2 mesh. The total power entering the B2 domain is equally split between the electron and ion heat channels. For the detachment analysis pure hydrogen plasma without impurities is used. To be able to have a fast model also the drift terms are inactive. The electron and ion densities are also prescribed at the CIB while the flux of the neutrals is set to zero. As boundary conditions at the wall side of the boundary, a radial decay length of $1 \mathrm{~cm}$ for the densities and temperatures of the ions and electron is used. The parallel flow velocity is assumed to be zero. The same boundary conditions as those at the wall side of the boundary are imposed on the walls in the private flux regions. Finally, the sheath condition is used at the divertor plate.

As for parallel transport, classical transport coefficients have been used. With respect to cross-field transport, there still exist relatively large uncertainties. Matching of experiment decay lengths at the midplane for old ASDEX, anomalous perpendicular transports with constant heat diffusivity $\chi_{e}=\chi_{i}=2 \mathrm{~m}^{2} / \mathrm{s}, \mathrm{D}=1 \mathrm{~m}^{2} / \mathrm{s}$ were used ${ }^{[6]}$. Considering the wall pumping effect, the effective recycling coefficient $\mathrm{R}$ of the wall is given by 0.99 for all the parts of the vacuum vessel.

\section{Results of simulation and experiment}


The dependence of the peak density and temperature at the target plates has been studied by means of a density scan at the CIB from $10^{17} \mathrm{~m}^{-3}$ to $10^{20} \mathrm{~m}^{-3}$ to identify the different divertor operational regimes. A summary of this edge density scan is given in Fig.2 for the SN/DN/modify SN configuration, respectively. The integral parallel particle and energy fluxes in $\mathrm{SN}$ configuration reaching the target plates as a function of the electron density at the CIB are shown in Fig.3. Divertor heat and particle balances for the outer divertor during SN configuration are shown in Figs. 4 where the fluxes into the outer divertor, at the target plate and integrated sources and integrated divergence of radial fluxes in each flux tube which starting from the target plate going backward to the X-point are analyzed.

When the electron density at the CIB is rather low $\left(n_{e} \leq 5 \times 10^{18} \mathrm{~m}^{-3}\right.$ in single null and $n_{e} \leq 2 \times 10^{19} \mathrm{~m}^{-3}$ in double nulls), the maximum electron density in the inner/outer divertor targets, either in the SN, DN or modify_SN configuration, rises linearly with the density at the CIB. The electron temperature decreases rapidly. This reflects the low recycling operational scenario which is often called linear or sheath-limited regime. But even in the linear case, quite large particle and energy losses from interaction with neutrals exists and radial fluxes are driven resulting in a broadening of the profiles which are shown in fig4 (left). It looks like the divertor throats of HL-2A are transparent, as indicated by the leakage of particles from the outer divertor into the main chamber

When the density at the CIB reaches intermediate values $\left(5 \times 10^{18}<n_{e} \leq 2 \times 10^{19} \mathrm{~m}^{-3}\right.$ in single null and $2 \times 10^{19}<n_{e} \leq 6 \times 10^{19} \mathrm{~m}^{-3}$ in double nulls), the difference between the maximum electron density at target plate and the mid-plane density becomes larger and then the maximum density approaches the mid-plane density while the electron temperature at the 
target decreases to $5 \mathrm{eV}$. This is not a local high recycling regime with large flux amplifications and much higher density at target plate than that at midplane. Compared with the modify SN configuration, the long divertor throat is shorten to form a modified SN divertor configuration simply. The density scan (fig.2, right) shows the expected high recycling features of the higher values of divertor to midplane densities. Fig.4 (middle) shows the particles entering into the divertor are not negative anymore but very small, while a large increase of the radial particle flux is observed mainly driven by large particle sources from neutrals spread out radially. This effect is getting even more pronounced and less and less energy and particle fluxes are reaching the plate compared with the scenario in the linear case.

Pushing the density at the CIB further in three configurations, the electron density at the inner target plate saturates while the temperature maximum decreases to less than $5 \mathrm{eV}$. Then it quickly induces the divertor detaches in the inner divertor first. At the highest density, detachment shows up with more and more negative neutral particle sources due to volume recombination in fig.4 (right). In addition, the particle fluxes into the outer divertor increases significantly, probably driven by the sources in the main chamber. As can be seen in Fig. 4, at the highest density, very close to the onset of a Marfe, the energy flux into the divertor is strongly reduced with practically no power reaching the plate. In this case, the plasma entered into the detachment regime.

The integral ion particle flux reaching the target plate in SN (Fig.3) now shows the expected signature in contrast to the local quantities discussed before, a linear rise at the beginning and then a drop after the transition to detachment. The power reaching the plate keeps linearly decreasing with the density at the CIB. 
The previous results can be easily understood from geometrical arguments, because the detachment characteristic of HL-2A is determined by the thin divertor throats. These are transparent for neutrals, but still quite well baffled towards the main chamber due to the long baffle lengths, resulting in momentum losses from the interaction of the streaming divertor plasma with this neutral cushion already at low densities. This resembles the fact that in HL-2A the validity of the traditional 2-point model and its regimes is no longer fulfilled, because the radial losses are always important and within a local analysis the high recycling phase does not show up, similar to island divertor in stellarator. However, in integral quantities the non-linear scaling of this regime reappears quite naturally. This shows the importance of the radial broadening due to the neutrals in the divertor throats. Fig.5 is one typical SN configuration discharge at HL-2A experiment. The plasma detaches at lower core density with the closed divertor, as predicted by SOLPS5.0. With the formation of a X-point and a divertor, the $\bar{n}_{e}$ goes up to $20 * 10^{18} \mathrm{~m}^{-3}$ (2nd line) at about $510 \mathrm{~ms}, n_{\text {targ } e t}$ near the strike point at the target plate $(\mathrm{z}=-87 \mathrm{~cm})$ increases to $20 * 10^{17} \mathrm{~m}^{-3}$ and $T_{\text {target }}$ decreases to lower than $10 \mathrm{eV}$ (4th line). $T_{\text {target }}$ at most other parts of the target plate is lower to $5 \mathrm{eV}$ (5th line). It shows that the most part of the area in outer target plate enters into partial detachment. At the same time, The Ha signals near the x-point (6th line) and the divertor chamber (8th line) rise while the Ha at upper $\mathrm{x}$-point area (7th line) keeps constant. It means an increase of the neutral hydrogen atom recycling near the $\mathrm{x}$-point area and divertor chamber. This is dominated by neutrals from the divertor chamber, because the Ha level at the upper x-point area is not changing. The increase of Ha is possible by the onset of volume recombination. 


\section{Conclusion}

HL-2A is characterized by its specific geometry with narrow and transparent divertor fans. The neutral recycling is effective to the divertor parameters. HL-2A with its closed divertor geometry has very good baffling of neutrals due to the long divertor baffles. However, the very thin divertor structures cause problems of getting power into it and onto the divertor plates. Neutral momentum losses exist from lower edge densities; it results in radial spreading of pressure and power. Therefore, the local 2-point model scaling is not observed and no high recycling regime shows up. Experiment results also show that the divertor easily enters into partially detachment at lower core density. This is very similar to island divertor in stellarator. 


\section{Figure Captions:}

Fig1. HL-2A SN (left), DN (middle) and modify-SN (right) grids as produced by the automatic grid generator.

Fig2. The decreasing peaked $\mathrm{Te}(\mathrm{eV})$ and increasing ne $\left(\mathrm{m}^{-3}\right)$ at midplane separatrix and target plates with the increasing CIB electron density ne $\left(\mathrm{m}^{-3}\right)$ scan. Here, nesepm/nemxip/nemxap are the peaked ne $\left(\mathrm{m}^{-3}\right)$ at midplane/inner/outer divertor, tesep/temxip/temxap are the maximal Te (eV) at midplane/inner/outer divertor for SN (left), DN (middle), modify SN (right) configurations, $\mathrm{P}_{\mathrm{CIB}}=500 \mathrm{~kW}, \mathrm{Bt}=2.5 \mathrm{~T}, D_{n}^{a}=1.0 \mathrm{~m}^{2} / \mathrm{s}, \chi_{e}=\chi_{i}=2 \mathrm{~m}^{2} / \mathrm{s}$.

Fig3. The integral parallel heat (W) and partial fluxes $\left(\mathrm{s}^{-1}\right)$ arriving at the target plate as a function of the CIB electron density $\left(\mathrm{m}^{-3}\right)$ in the HL-2A SN configuration.

Fig4. Radial outer divertor particle flux $\left(\mathrm{s}^{-1}\right)$ and the energy flux (W) balance for CIB electron density ne $\mathrm{CIB}_{\text {B }}$ of $5 \times 10^{17} \mathrm{~m}^{-3}$ (left), $8 \times 10^{18} \mathrm{~m}^{-3}$ (middle), $2.5 \times 10^{19} \mathrm{~m}^{-3}$ (right) in HL-2A SN configuration, $\mathrm{P}_{\mathrm{CIB}}=500 \mathrm{~kW}$. Separatrix is between 6 and 7 .

Fig5. A typical SN divertor configuration discharge on HL-2A. From the top, there is the plasma current Ip (kA), line-averaged density $\bar{n}_{e}\left(10^{18} \mathrm{~m}^{-3}\right)$, Gas puffing evolution. $n_{t \text { arget }}\left(10^{17} \mathrm{~m}^{-3}\right)$ and $T_{t \text { arget }}(\mathrm{eV})$ in the 4 th and 5 th are the electron densities and temperatures at the outer target plate of $\mathrm{z}=-87 \mathrm{~cm}$ and $-82 \mathrm{~cm} . \mathrm{H}_{a}$ chord signals pass through $\mathrm{X}$-point, edge plasma (upper X-point) and divertor chamber are shown in 6th, 7th, 8th lines. 

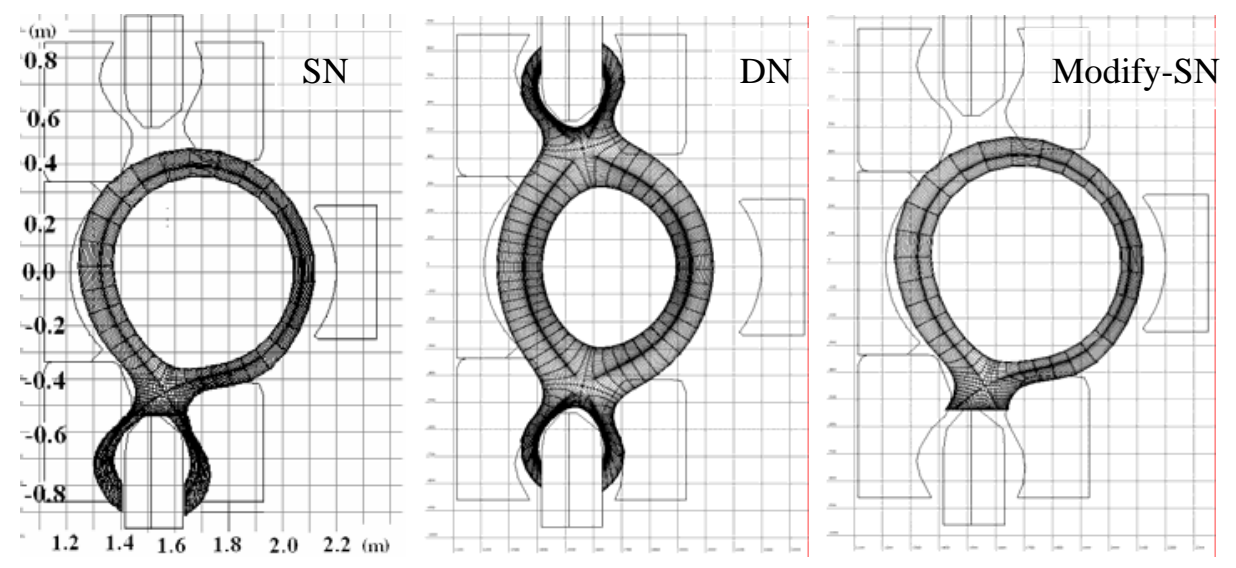

Fig.1. 

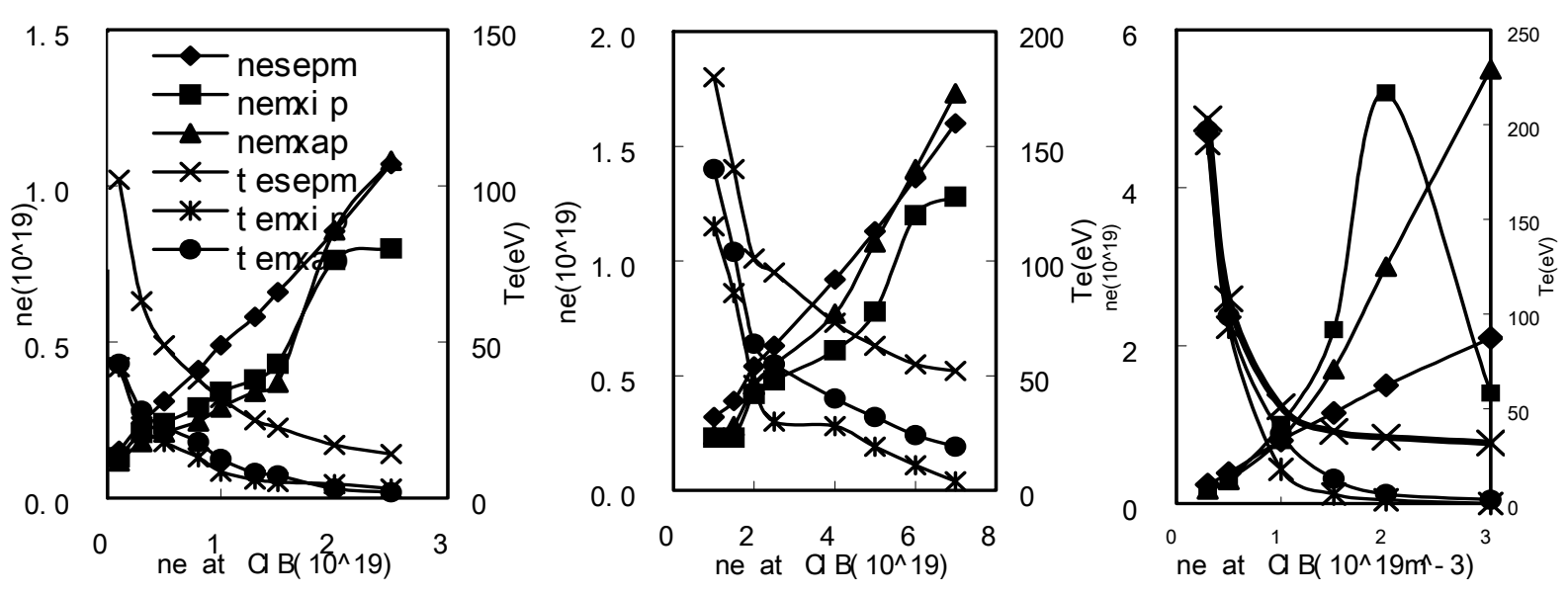

Fig.2. 


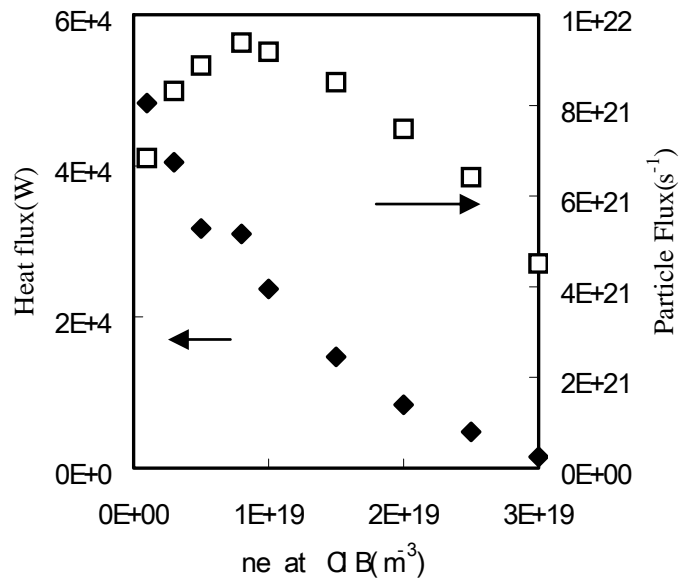

Fig.3. 

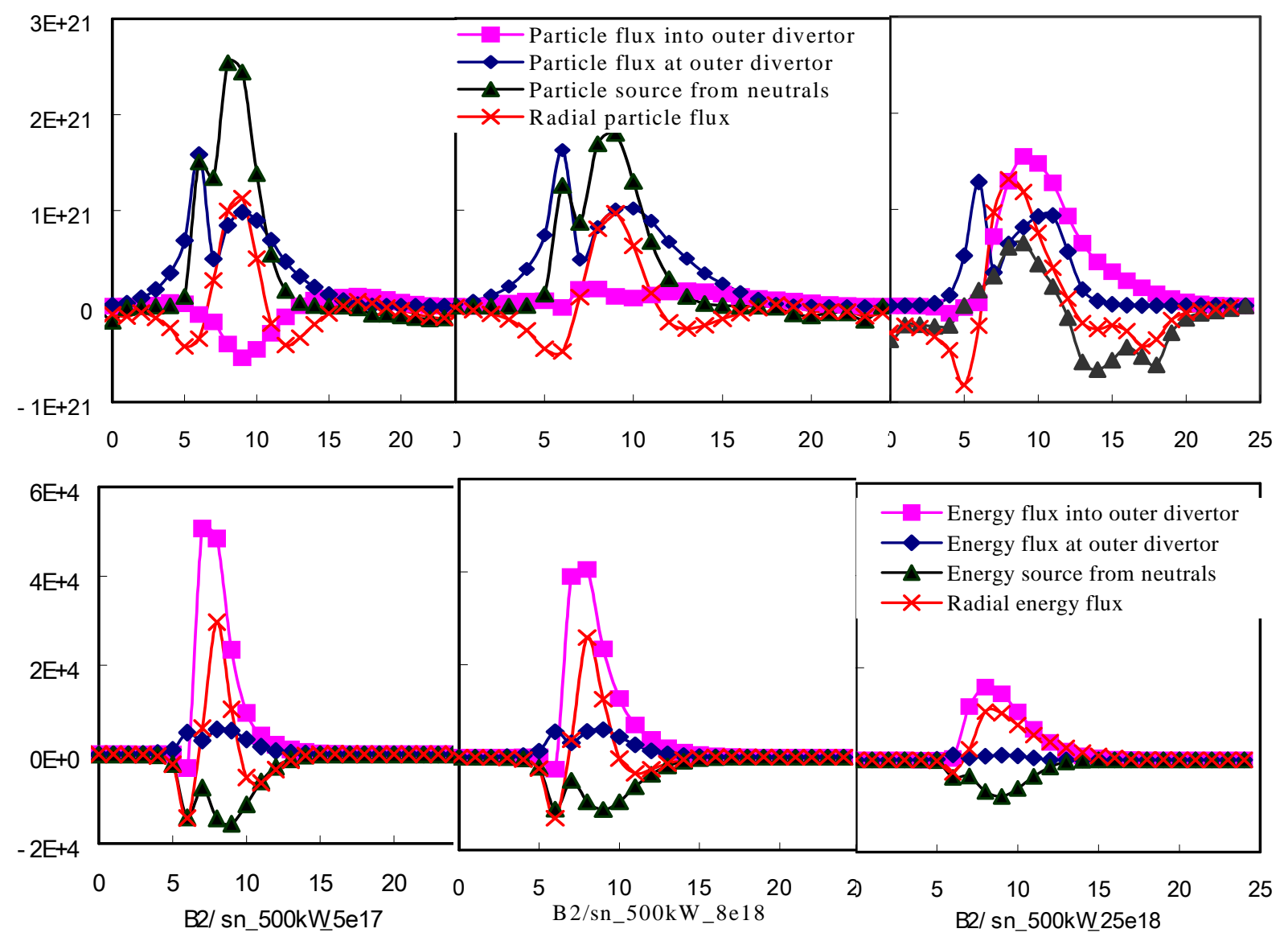

Fig.4. 

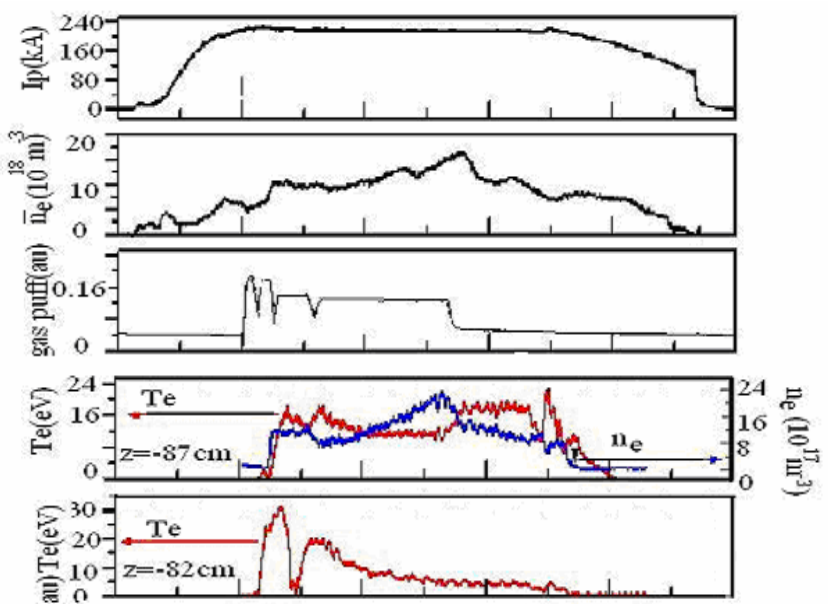

兽 $10-z=-82 \mathrm{~cm}$

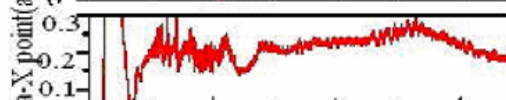

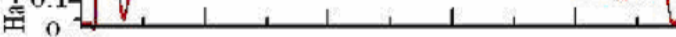

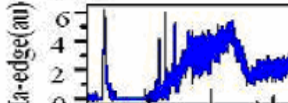

空 0 ,

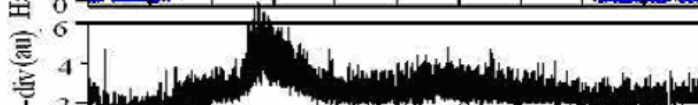

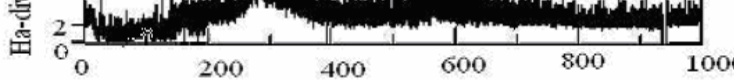

t(ms)

Fig.5. 


\section{References:}

${ }^{[1]}$ R. Schneider et al., J. Nucl. Mater. 196-198, (1992)810.

${ }^{[2]}$ D.Reiter, J.Nucl.Mater.196-198, (1992) 80.

${ }^{[3]}$ D.P.Coster, et al., 28th EPS conference on contr.Fusion and plasma phys. ECA, Vol.25, (2001) 1601.

${ }^{[4]}$ X. Bonnin, R. Schneider, D. Coster, V.A. Rozhansky and S.P. Voskoboynikov, J. Nucl. Mater. 290-293: (2001) 829.

${ }^{[5]}$ D. P.Coster,X.bonin,B.Braams, et al., Proc.19th IAEA Fusion Energy Conference,Lyon,2002,TH/P2-13.

${ }^{[6]}$ J.Neuhauser, et al, Plasma Phys. Control. Fusion 31, (1989) 1551. 